\title{
Characterization of genetically targeted neuron types in the zebrafish optic tectum
}

\author{
Estuardo Robles $^{1}{ }^{*}$, Stephen J. Smith ${ }^{2}$ and Herwig Baier ${ }^{1}$ \\ 1 Department of Physiology, University of California San Francisco, San Francisco, CA, USA \\ 2 Department of Molecular and Cellular Physiology, Stanford University, Stanford, CA, USA
}

\section{Edited by:}

Abdel El Manira, Karolinska Institute, Sweden

\section{Reviewed by:}

Hitoshi Okamoto, RIKEN Brain Science Institute, Japan

Florian Engert, Harvard University, USA Abhinav Grama, Harvard University, USA

\section{*Correspondence:}

Estuardo Robles, Department of Physiology, University of California San Francisco, 1550 Fourth Street, San Francisco, CA, USA. e-mail: estuardo.robles@ucsf.edu
The optically transparent larval zebrafish is ideally suited for in vivo analyses of neural circuitry controlling visually guided behaviors. However, there is a lack of information regarding specific cell types in the major retinorecipient brain region of the fish, the optic tectum. Here we report the characterization of three previously unidentified tectal cell types that are specifically labeled by $\mathrm{dl} \times 5 / 6$ enhancer elements. In vivo laser-scanning microscopy in conjunction with ex vivo array tomography revealed that these neurons differ in their morphologies, synaptic connectivity, and neurotransmitter phenotypes. The first type is an excitatory bistratified periventricular interneuron that forms a dendritic arbor in the retinorecipient stratum fibrosum et griseum superficiale (SFGS) and an axonal arbor in the stratum griseum centrale (SGC). The second type, a GABAergic non-stratified periventricular interneuron, extends a bushy arbor containing both dendrites and axons into the SGC and the deepest sublayers of the SFGS. The third type is a GABAergic periventricular projection neuron that extends a dendritic arbor into the SGC and a long axon to the torus semicircularis, medulla oblongata, and anterior hindbrain. Interestingly, the same axons form en passant synapses within the deepest neuropil layer of the tectum, the stratum album centrale. This approach revealed several novel aspects of tectal circuitry, including: (1) a glutamatergic mode of transmission from the superficial, retinorecipient neuropil layers to the deeper, output layers, (2) the presence of interneurons with mixed dendrite/axon arbors likely involved in local processing, and (3) a heretofore unknown GABAergic tectofugal projection to midbrain and hindbrain. These observations establish a framework for studying the morphological and functional differentiation of neural circuits in the zebrafish visual system.

Keywords: visual system, synapse, neural circuit, array tomography, GABAergic, laminar targeting

\section{INTRODUCTION}

The larval zebrafish visual system has emerged as a valuable system in which to examine how neural circuits transform sensory information into motor commands that initiate stereotyped behaviors such as the optokinetic and optomotor responses. The primary retinorecipient brain area in fish is the optic tectum, which mediates complex, visually evoked behaviors such as prey capture (Gahtan et al., 2005). The optic tectum is a multilaminated structure with a dense neuropil in which tectal cell dendrites receive synapses from sensory afferents and tectal interneurons (reviewed by Meek, 1983). Studies examining structural differentiation and synapse formation in tectal neurons have revealed general mechanisms of circuit formation, however there is a lack of information regarding specific cell types in the tectum. Functional imaging data obtained from tectal neurons has permitted a categorization of tectal neurons into broad classes based on their visual response properties (Niell and Smith, 2005). However, it is unclear whether visual response properties correlate with specific cell attributes such as neurite morphology, synaptic connectivity, or neurotransmitter phenotype. In order to understand circuit function in the tectum it is necessary to couple functional imaging data to specific neuron types. In the adult goldfish tectum a purely morphological cell type classification has revealed a high degree of diversity (Meek and Schellart, 1978). In the larval zebrafish tectum numerous studies have examined the axon pathfinding or synapse formation of retinal axons in the tectum, whereas relatively few studies have examined specific tectal neuron types. The identification of genetic markers that label specific tectal cell types will reveal molecular mechanisms that generate neuronal diversity and serve as valuable tools with which to examine the structure and function of specific neuron types within complex neural circuits.

Recent studies have begun to characterize the molecular and morphological diversity of neurons in the larval zebrafish tectum. Enhancer trapping is a powerful technology that enables molecular characterization of neuron types labeled by random insertion of a reporter transgene in the genome. A recent Gal4 enhancer trap conducted in zebrafish generated 184 stable transgenic lines with unique expression patterns in the larval nervous system (Scott et al., 2007). Of these lines, 13 had unique expression patterns in the tectum and three lines were subsequently surveyed for morphological cell types using a genetic mosaic labeling technique (Scott and Baier, 2009). This study identified a variety of neuronal morphologies that correspond well with neuron classes previously identified in the adult goldfish. Grouping neurons into categories based on cell body position and morphology revealed that these general classes of neurons are differentially labeled in the three ET lines examined. 
This suggests that the enhancer elements targeted in these lines confer specific structural features, likely as part of a complex combinatorial code that ultimately specifies unique cell types. However these groupings were based solely on morphological criteria and therefore these classes may consist of distinct cell types with divergent connectivities and/or neurotransmitter phenotypes.

An alternative approach is to use candidate promoters derived from genes expressed in the tectum to label subpopulations of tectal neurons. Okamoto and colleagues recently characterized a brn3a (pou4f1):GFP reporter as a marker for roughly 50\% of tectal neurons in addition to labeling subsets of cells in the eye, pretectum, habenula, and torus semicircularis (TS; Sato et al., 2007). Given the large number of tectal cell types identified in the adult goldfish (Meek and Schellart, 1978), this transgene likely labels many different cell types in the larval zebrafish. This was confirmed by single-cell labeling using a transient expression strategy that allowed detailed morphological examination of brn3a: $g f p$-positive neurons. Although several different tectal neuron types were observed in this study, thorough characterization was restricted to a population of projection neurons with efferent axons that project ipsilaterally to the hindbrain. Morphological analysis of this cell type revealed a high degree of variability in both the size of the dendritic arbor and the termination site of the descending axonal projection, suggesting that this constitutes a broad, heterogeneous population of neurons. Together these studies confirm the large diversity of neuronal cell types in the larval zebrafish tectum and have begun to reveal fundamental organizing principles in the optic tectum.

Although these studies shed light on cell type diversity in the larval tectum, both studies utilized transgenes with broad expression patterns. This presents a formidable challenge to precisely determining the complement of cell types labeled by the transgene, and may lead to rare cell types going uncharacterized. Furthermore, classification of cells into broad categories based on neurite projection patterns cannot identify subcategories defined by differences in synaptic connectivity or molecular traits such as neurotransmitter phenotype. An alternative to these approaches is to identify transgenes that label small subsets of cells, and therefore fewer neuron types. Here we report the characterization of three previously unidentified tectal cell types that are specifically labeled by a construct containing the distal-less six $(d l x 6) 5$ promoter and a fragment of the intergenic region situated between $d l \times 5 / 6$. Live imaging and retrospective array tomography of genetically targeted neurons in the larval tectum consistently labeled three cell types that differed in morphology, synaptic connectivity, and neurotransmitter phenotype. The ability to genetically target small subpopulations of neurons is essential for cell type-specific analyses of neuronal differentiation and function within the developing visual system.

\section{MATERIALS AND METHODS TRANSGENIC FISH LINES}

The $\operatorname{Tg}(1.4 d l x 5 a-d l x 6 a: G F P)$ ot1 fish line has been previously described (Ghanem et al., 2003) and was generously provided by the laboratory of Dr. Marc Ekker (University of Ottawa). Pou4f3:gfp transgenic line has been described (referred to as TgBrn3c:GAP43GFP $^{356 t}$ in Xiao et al., 2005). Maintenance and spawning of adult zebrafish was performed as described by Westerfield (2000). All procedures were approved by the Animal Care and Use Committees of both Stanford University and UCSF.

\section{PLASMID CONSTRUCTION}

The $d g 4$ ii plasmid was generated using a dlx5/6:GFP:I56ii plasmid generously provided by the laboratory of Dr. Marc Ekker (University of Ottawa). The GFP in this plasmid was replaced with the coding region for Gal4-VP16 from the alpha1-tubulin:Gal4-VP16 plasmid (Koster and Fraser, 2001). The following plasmids have been previously described: uas:egfp (Jontes et al., 2004); uas:dsred-express (Niell et al., 2004); uas:dsred/uas:synaptophysin-gfp (Meyer and Smith, 2006).

\section{MICROINJECTION OF ZEBRAFISH EMBRYOS}

DNA was pressure-injected into 1-4 cell-stage embryos. Both Gal4-VP16 driver and UAS reporter plasmids were injected at a concentration of 20-25 $\mu \mathrm{g} / \mathrm{ml}$ in Danieau's solution. Embryos were raised at $28^{\circ}$ in medium containing $0.2 \mathrm{mM}$ phenylthiourea to block pigment formation. At 3-5 dpf healthy embryos were embedded in low melting-point agarose and screened for fluorescent reporter expression in the tectum.

\section{IN VIVO IMAGING}

Imaging of EGFP expression was performed using a custom built two-photon microscope (Niell et al., 2004). Excitation was provided by a Mira 900 Ti:Sapphire laser tuned to $820 \mathrm{~nm}$. Multicolor imaging was performed on a Zeiss Pascal confocal microscope equipped with a multi-line Ar laser for GFP excitation (488 nm) and a red He:Ne laser for dsRed (546 nm). On both microscopes optical sections were acquired using $1 \mu \mathrm{m} z$-steps for interneurons and 1-2 $\mu \mathrm{m}$ z-steps for projection neurons. Image stacks were visualized and analyzed using ImageJ software $(\mathrm{NIH})$; volumetric renderings were made with the 3D Viewer plugin written by Benjamin Schmid (Würzberg University). Measurements of neurite arbors were performed using the $\mathrm{z}$-stacks acquired from larvae mounted dorsal side up. Skin autofluorescence was used as a marker for the surface of the tectal neuropil. Reticulospinal circuitry was visualized in paraformaldehyde-fixed embryos by pressure injection of a 1\% solution of the lipophilic dye DiI into the spinal cord at the level of the anus and incubated $24 \mathrm{~h}$ at $23^{\circ} \mathrm{C}$ for dye diffusion.

\section{ARRAY TOMOGRAPHY}

Zebrafish larvae were prepared for array tomography as described by Micheva and Smith (2007), with the following modifications. Following fixation in PBS containing 4\% Paraformaldehyde and graded ethanol dehydration, larvae used for $1 \mu \mathrm{m}$ thick sections were embedded in soft-grade LR-White resin (SPI supplies). Following polymerization in gelatin capsules resin blocks were sectioned at a thickness of either $200 \mathrm{~nm}$ or $1 \mu \mathrm{m}$ on a Leica EM-UC6 ultramicrotome and adhered onto glass microscope slides. Arrays were imaged using custom software for semi-automated acquisition, aligned in ImageJ, and 3D-rendered with Zeiss Axiovision software. Mouse anti-SV2 antibody was obtained from the Developmental Studies Hybridoma Bank (University of Iowa). Rabbit anti-GABA polyclonal antibody was purchased from Chemicon (AB131). Cells were scored as GABA-positive if the immunofluorescence intensity of 
the cell body was greater than $3 \times$ the intensity of GABA-negative skin cells. Colocalization of GFP and SV2 was conducted using a custom ImageJ plugin developed by Brad Busse.

\section{FLUORESCENCE IN SITU HYBRIDIZATION}

vglut2b in situ probe has been previously described (Smear et al., 2007). Fluorescence in situ hybridization was performed using DIGlabeled probe followed by labeling with an anti-DIG-POD antibody (Roche). Antibody signal was amplified using tyramide signal amplification (TSA-plus Cy3 system, Perkin-Elmer). Anti-chicken GFP antibody was purchased from GeneTex. Due to variability in the quality of in situ hybridization labeling analysis was restricted to batches of embryos in which the signal strength was sufficient to allow clear discrimination between vglut $2 b$-positive and negative cell bodies. This variability made it unfeasible to perform vglut $2 b$ in situ analysis in the small percent of embryos with individually labeled neurons as identified by confocal microscopy.

\section{RESULTS \\ A $d / x 5 / 6$ STABLE TRANSGENIC LABELS SPARSE CELL POPULATIONS THROUGHOUT THE LARVAL BRAIN}

The $\operatorname{Tg}(1.4 d l x 5 a-d l x 6 a: G F P)$ ot1 line contains a transgene with GFP flanked by both a $3.5-\mathrm{kb}$ fragment of the $d l x 6$ promoter and a $1.4-\mathrm{kb}$ fragment of the $d l x 5 / 6$ intergenic region (Ghanem et al., 2003). This stable transgenic line will herein be referred to as $d l x 5 / 6: G F P$. This $1.4 \mathrm{~kb}$ fragment of the intergenic region contains two cis-acting regulatory elements, I56i and I56ii, which are thought to define molecularly distinct populations of cortical interneurons in the mouse (Ghanem et al., 2008). In order to begin to characterize cell types labeled by $d l \times 5 / 6$ transgenes in the zebrafish tectum we conducted live two-photon laser-scanning microscopy of $d l x 5 / 6$ :GFP larvae. Live imaging revealed a large percentage of cells labeled by GFP in the telencephalon, whereas sparser cell labeling was observed in the tectum, cerebellum, and diencephalon (Figures 1A-C). In the tectum we estimate that these GFP-positive cells represent 5-10\% of all tectal neurons and are distributed sparsely throughout both the neuropil and stratum periventriculare (SPV), which contains the majority of tectal neuron cell bodies (Figure 1A).

\section{THE MAJORITY OF $d / x 5 / 6$-LABELED TECTAL CELLS ARE GABAERGIC}

Expression of Dlx family proteins has been shown to be a strong determinant of GABAergic specification in mouse cortical neurons (Stuhmer et al., 2002). Similarly, the $d l x 5 / 6$ :GFP transgenic in this study has been used to study GABAergic interneurons in the zebrafish olfactory bulb (Mack-Bucher et al., 2007). To determine the percentage of $d l x 5 / 6: G F P$-positive tectal neurons that are GABAergic we modified array tomographic techniques for thinsectioning of resin-embedded tissues (Micheva and Smith, 2007) in order to generate arrays of $1 \mu \mathrm{m}$ thick sections. Use of array tomographic methods allowed for uniform antibody staining of superficial and deep brain regions. These thicker sections facilitated reconstruction of the entire larval brain while providing single-cell resolution for neurotransmitter analysis. Immobilized arrays of $1 \mu \mathrm{m}$ thick sections were subsequently stained with an antibody that recognizes the neurotransmitter GABA and labeled with the fluorescent nuclear marker DAPI.
Three-dimensional reconstruction of array images allowed us to visualize the distribution of GABAergic cells throughout the entire brain volume (Figures 2A-D). Analysis of GABA immunofluorescence indicated that $85.4+5.3 \%$ of GFP-positive neurons in the tectum are GABAergic ( 969 of 1,135 cells from 5 tecta). To determine the spatial distribution of GABA-positive $d l x 5 / 6$ : GFP cells we divided the tectum into three regions: the SPV layer that contains most tectal neuron cell bodies, the main neuropil layer (NP), and a superficial neuropil layer (Sup), which is directly beneath the skin that covers the tectum (Figure 2E). Subregion analysis revealed that $100 \%$ of GFP-positive neurons with cell bodies in the Sup were also positive for GABA immunofluorescence, whereas a small percentage of GFP-positive neurons in the NP and SPV layers were not GABA immunoreactive. This suggests that at least one $d l x 5 / 6$ :GFPpositive cell type is not GABAergic.

Several studies have previously reported the presence of glutamatergic, GABAergic, and cholinergic neurons in the larval tectum. Notably, studies have also reported an absence of aminergic (noradrenergic and serotonergic) and glycinergic neurons at this developmental stage (Higashijima et al., 2004; McLean and Fetcho, 2004). Immunostaining and in situ hybridization data suggest that a large percentage of tectal neurons are glutamatergic or GABAergic (Higashijima et al., 2004; Sato et al., 2007; Smear et al., 2007), whereas cholinergic cells are relatively rare (Arenzana et al., 2005). To determine if the non-GABAergic population of neurons labeled in dlx5/6:GFP larvae are glutamatergic we conducted $v g l u t 2 b$ fluorescence in situ hybridization in conjunction with retrospective GFP antibody staining. vglut2b in situ analysis revealed that a large percentage of cells in the SPV layer of the tectum are glutamatergic (Figure 3A). Subregion analysis of fluorescence in situ labeling confirmed that GFP-positive neurons with cell bodies in the superficial neuropil, which are GABA-positive, do not express vglut $2 b$ (Figure 3B). In contrast, a small percentage of dlx5/6:GFP-positive neurons in the SPV layer were positive for vglut $2 b$ cDNA (Figure 3C; $15.6+1.5 \%$; data obtained from 555 cells from nine different larva). Closer examination of vglut $2 b$ positive neurons confirmed the presence of discrete perinuclear puncta within the cell body (Figure 3B). These results indicate that the non-GABAergic population of tectal neurons labeled in dlx5/6:GFP larvae is glutamatergic.

\section{A $d / x 5 / 6$ TRANSGENE FOR MOSAIC GENETIC LABELING OF TECTAL CELL TYPES}

To label a small subset of tectal neurons we employed a mosaic labeling technique. We focused on transgene constructs containing the $d l x 5 / 65^{\prime}$ promoter and only one of the two known intergenic enhancer elements ( $156 i$ or I56ii). Based on preliminary findings that a $d l x 5 / 6$ construct containing the $156 i$ enhancer does not drive transgene expression we utilized a construct containing the $d l x 65^{\prime}$ promoter and the I56ii enhancer region. Previous studies have demonstrated that broad Dlx expression patterns can be subdivided based on the activity of individual enhancer elements (Ghanem et al., 2008). This means that cells labeled with a $d l x 5 / 6$ construct containing only the I56ii enhancer most likely represent a subset of the neurons labeled in transgenic dlx5/6:GFP larvae. The Gal4-UAS expression system has been used extensively and promotes strong reporter gene expression and in our experience reduces the number of cells labeled 

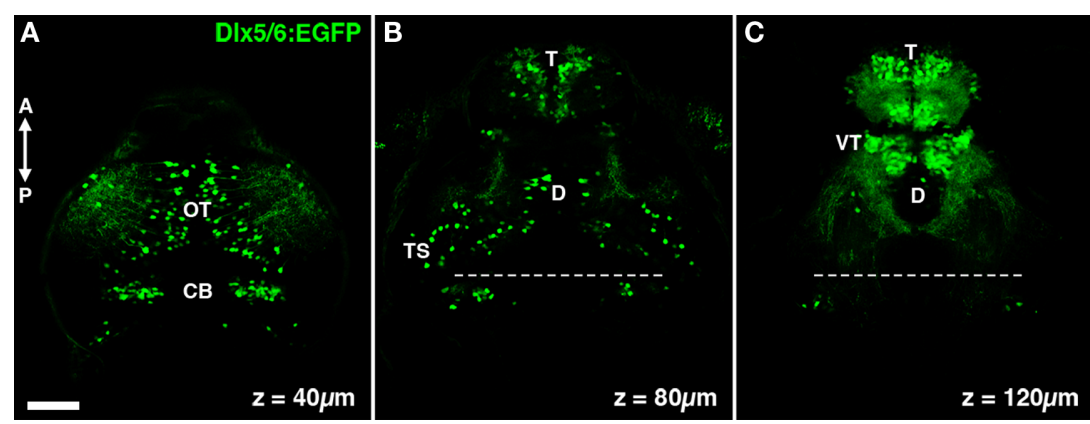

FIGURE 1 |The dlx5/6:GFP transgene labels specific neuronal populations in the larval zebrafish brain. (A) A single confocal section acquired $40 \mu \mathrm{m}$ below the dorsal surface of the brain. GFP transgene expression is observed in cells in the optic tectum (OT) and cerebellum (CB). (B) At a depth of $80 \mu \mathrm{m}$ dense GFP labeling of cells is seen in the olfactory bulb of the telencephalon (T), in addition to sparse labeling throughout the diencephalon (D) and mesencephalic structures such as the torus semicircularis (TS). (C) At a depth of $120 \mu \mathrm{m}$ labeling is largely restricted to the telencephalon and the ventral thalamus (VT). Dashed line indicates midbrain-hindbrain boundary. Scale bar, $50 \mu \mathrm{m}$.
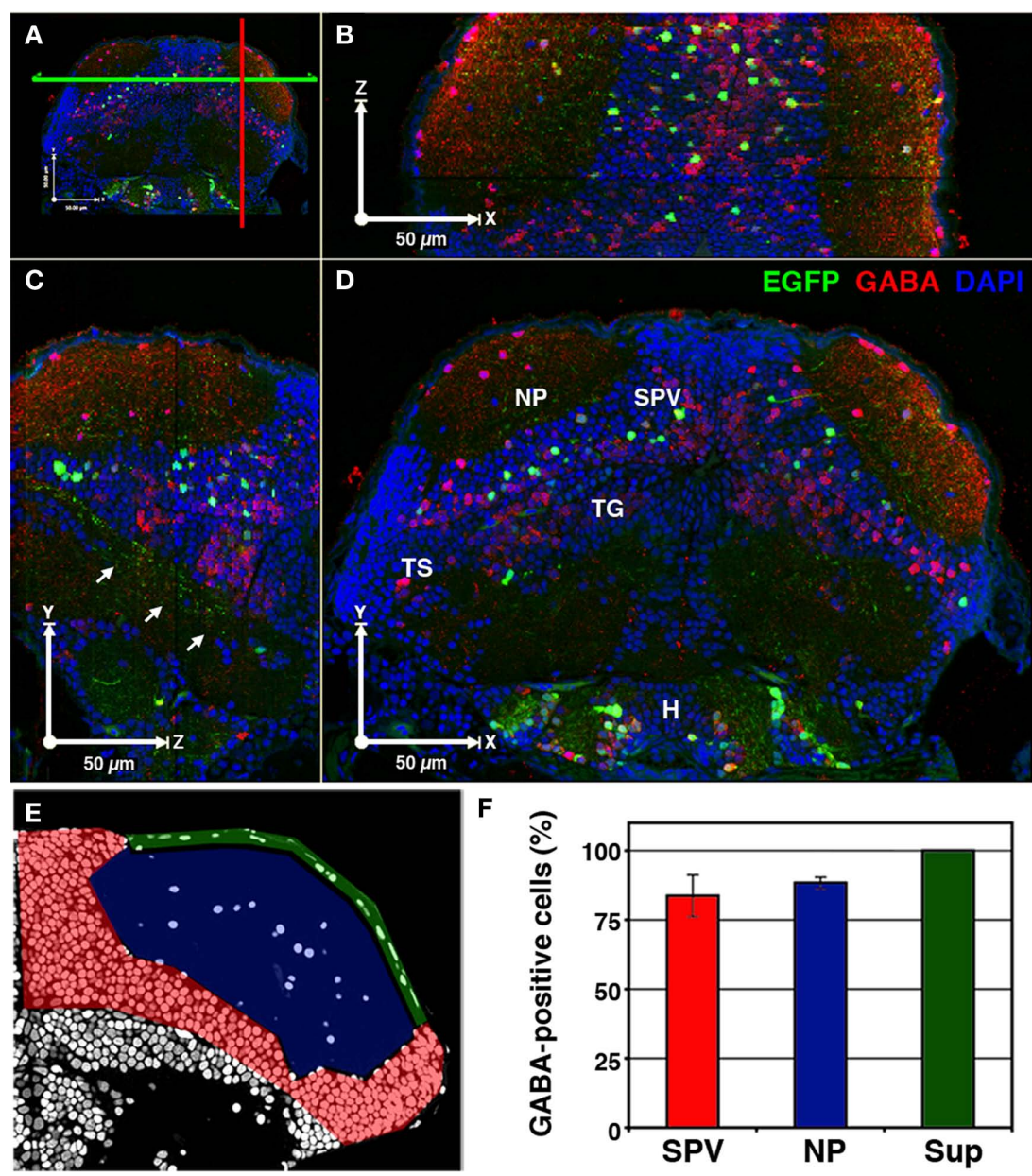

FIGURE 2 |A majority of cells labeled in dlx5/6:GFP larvae are GABAergic. $3 \mathrm{D}$ reconstruction of a 5-dpf $d / x 5 / 6$ : GFP larvae that was fixed and sectioned at a thickness of $1 \mu \mathrm{m}$ and labeled by GABA immunofluorescence and DAPI nuclear staining. (A) Colored lines indicate the relative locations of the cut-views presented in (B-D). (B) Cut-view in the $X Z$ plane (dorsal view). (C) Cut-view in the $Y Z$ plane (sagittal). (D) Cut-view in the $X Y$ plane (coronal). Note the single-cell resolution in $3 \mathrm{D}$ and the high density of GABAergic cells in the periventricular cell

layer (SPV) and neuropil (NP) regions of the tectum and the relative sparsity of GFP-labeled neurons. Arrows in (C) indicate axonal tracts of diencephalic and mesencephalic origins en route to the hindbrain. (E) A single section labeled with DAPI. Colored shading indicates the tectal regions defined for GABA phenotypic analysis: SPV (red), neuropil (blue), and superficial neuropil (green). (F) GABAergic phenotypic analysis in the optic tectum of $5 \mathrm{dpf} d l \times 5 / 6$ :GFP larvae. Tegmentum (TG), hypothalamus (H), torus semicircularis (TS). Scale bar, $50 \mu \mathrm{m}$ in (B-E). 

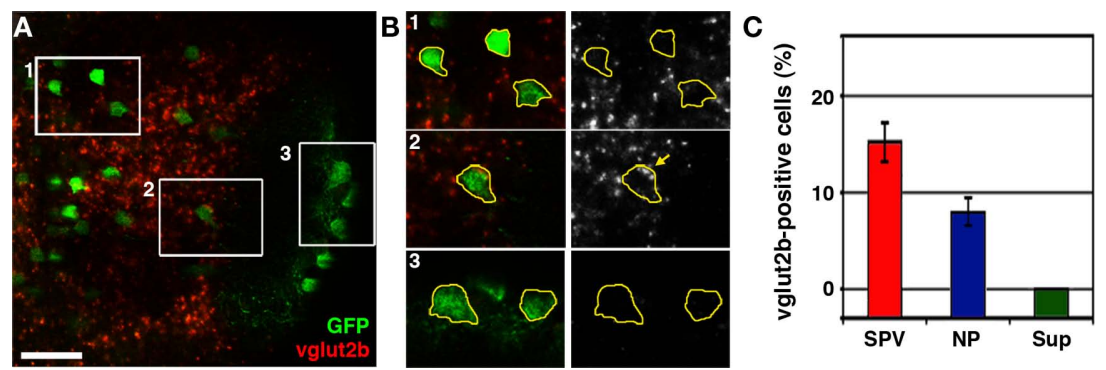

FIGURE 3 |The dlx5/6:GFP transgene labels a subpopulation of glutamatergic neurons. (A) Confocal image of vglut $2 b$ fluorescence in situ hybridization in a 5-dpf $d / x 5 / 6$ :GFP larvae. (B) $2 \times$ magnification of regions indicated by boxes in (A). Merged images of GFP immuofluorescence and vglut2b in situ labeling (left) and vglut $2 b$ in situ labeling alone (right). GFP fluorescence was used to trace cell body outlines. Note strong, punctate vglut2b in situ signal in the perinuclear region of cell body in B2. In contrast, the majority of cells in the SPV (B1) and all cells in the superficial NP (B3) lacked this punctate vglut $2 \mathrm{~b}$ mRNA signal. (C) Quantification of glutamatergic phenotypic analysis in dlx5/6:GFP. Scale bar, $20 \mu \mathrm{m}$ in (A), $10 \mu \mathrm{m}$ in (B). by DNA microinjection, possibly due to the requirement that both plasmids be incorporated into the same cell (Koster and Fraser, 2001). Therefore, we created a construct containing the coding region for a Gal4-VP16 fusion protein flanked by the $d l x 6$ promoter and the I56ii enhancer ( $d l x 5 / 6:$ gal4-vp16:I56ii, which we will refer to as $d g 4 i i)$. For initial experiments this plasmid was coinjected with a uas:egfp or plasmid into 1-4 cell-stage embryos, which were screened for tectal GFP expression between 3 and $4 \mathrm{dpf}$.

\section{CRITERIA FOR IN VIVO TECTAL CELL TYPE CLASSIFICATION}

Tectal cell types were characterized using the following criteria: cell body position, neuronal morphology, location of presynaptic specializations, and neurotransmitter phenotype. Live laser-scanning microscopy of tecta containing GFP or DsRed-labeled cells allowed rapid determination of cell body position and, in many cases, enabled a preliminary assessment of neurite morphology (Figure 4B). Volume rendering of confocal z-stacks was used to carefully examine neurite morphology with respect to the tectal neuropil layers, which are optimally visualized when an image volume obtained from a larva mounted dorsal side up is rotated approximately $45^{\circ}$ laterally (Figures 4C-E). To validate this approach a subset of embryos were labeled by coinjection of $d g 4 i i$ and a uas:dsred vector into transgenic pou4f3:GFP embryos, in which GFP is expressed by a subset of retinal axons that terminate in three layers of the tectal neuropil: the stratus opticum (SO) and the two deepest layers of the three-layered stratum fibrosum et griseum superficiale (SFGS; Xiao et al., 2005 and Figures 4A,E). As shown in Figure 4, a $45^{\circ}$ volume slice from one of these larvae reveals the laminar structure of an individual DsRed-labeled neuron and GFP-labeled pou4f3:gfp axons as markers for specific laminae. This analysis also provided us with relative thicknesses of the labeled neuropil layers during these developmental stages, which in combination with published findings allowed us to determine the laminar position of neurites in wild-type larvae. To examine the connectivity of identified cell types we determined the location of presynaptic specializations by using the $d g 4 i i$ construct to drive expression of cytosolic DsRed and a synaptophysin-GFP fusion protein using a bicistronic UAS construct (uas:dsred/uas:syp-gfp). This bicistronic construct has been used previously to simultaneously visualize neuronal morphology and presynaptic specializations in zebrafish neurons (Meyer and Smith, 2006).
Coinjection of both $d g 4 i i$ and uas:egfp plasmids allowed us to identify 92 individually labeled tectal neurons that were subsequently imaged by two-photon microscopy. Approximately $10 \%$ of injected embryos had cells labeled in the tectum, and of those $3-5 \%$ had individually labeled neurons that could be used for cell type analysis. Using morphological criteria obtained from image z-stacks we identified three distinct cell types that were consistently labeled using the $d g 4 i i$ construct: one class of projection neuron and two classes of tectal interneurons. Eighty-five of the 92 GFP-labeled neurons fell into one of these three categories. The remaining neurons had highly variable morphologies and may represent non-specific labeling, which is known to occur in transient expression studies.

\section{MORPHOLOGY AND CONNECTIVITY OF THE bSPVIN CELL TYPE}

Of the 92 cells individually labeled by the $d g 4 i i$ construct 32 were bistratified periventricular interneurons (bsPVINs), which have a very precise laminar morphology that helped to establish the aforementioned classification criteria. The cell bodies of these neurons were located in the deeper and intermediate layers of the SPV, suggesting these cells are absent from the shallow SPV adjacent to the neuropil. bsPVINs were also excluded from the anterior and lateral edges of the cell body layer (Figure 5D). The bsPVIN cell body gives rise to a single apical process that spans all but the most superficial neuropil layers (Figures 5B-C) and lacks an efferent process. Within the neuropil the bsPVIN neurite had a very characteristic stratified morphology with two distinct horizontal arborizations formed in different neuropil layers. Detailed analysis of brightly labeled bsPVIN arborization at $5 \mathrm{dpf}$ revealed that the deeper arborization was located 39.2 $\pm 2.3 \mu \mathrm{m}$ (SEM; $n=11$ ) from the dorsal skin overlying the tectal neuropil, which corresponds to the stratum griseum centrale (SGC; Figure 4E). The more superficial arborization was on average located $13.0 \pm 1.5 \mu \mathrm{m}(\mathrm{SEM} ; n=11)$ from the dorsal skin, which corresponds to the most superficial layer of the SFGS. This sublayer is innervated by retinal axons but not the retinal subpopulation marked by pou $4 f 3: g f p$ expression (Figure 4A). Visualization of bsPVIN arbors in the plane orthogonal to the synaptic layers, as shown in Figure 4C, revealed that both of these arbors were very precisely targeted. The average thickness of the SGC targeted arbor was $6.8 \pm 0.7 \mu \mathrm{m}(n=11)$ and the average thickness of the SFGS targeted arbor was $5.5 \pm 0.4 \mu \mathrm{m}(n=11)$. The width of the 

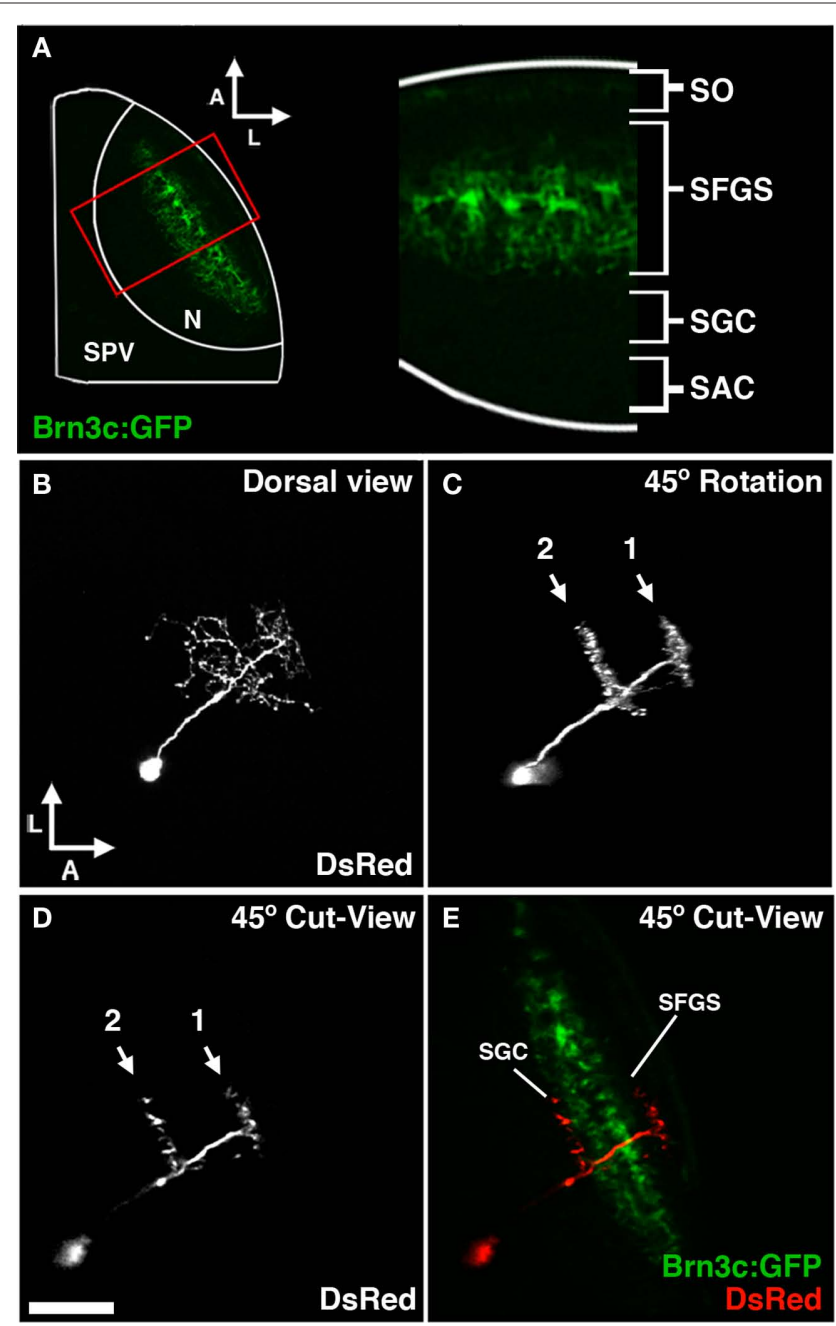

FIGURE 4 | Morphological analysis of neurons labeled by $\mathbf{d g} 4 \mathrm{ii}$ plasmid. (A) Approach used to determine laminar targeting of neurites in the tectal neuropil. Image of pou4f3:gfp fluorescence, which labels a subset of RGC axons that target the SO and the two deepest sublayers of the SFGS. This signal was used to determine the relative thickness of the SO and SFGS layers, which receive the vast majority of retinal afferents. (B) Maximum projection of a confocal image volume obtained from a single tectal neuron at $5 \mathrm{dpf}$ labeled by blastomere injection of $d g 4 i i$ and uas: $d s r e d$ plasmids. Note the single apical neurite that gives rise to a compact arbor lacking an efferent projection. (C) Forty-five degree rotation of 3D volume rendering performed on image stack in $\mathrm{B}$ reveals precise stratification of two distinct arbors. (D) Cut-view at the same $45^{\circ}$ orientation as in (C) demonstrates the roughly $45^{\circ}$ orientation of the apical dendrite. (E) A merged image of cut-view in (D; red) and pou4f3:gfp fluorescence (green) reveals that the superficial arbor targets the SFGS layer, whereas the deeper arbor targets the SGC layer below the SFGS. Scale bar, $50 \mu \mathrm{m}$ in (A), $25 \mu \mathrm{m}$ in (B-E).

bsPVIN arbors in the plane of the neuropil layers revealed that the deeper SGC arbor is significantly wider than the more superficial SFGS arbor, which may have important implications for visual processing in the tectum.

The specific laminar targeting exhibited by the bsPVIN class of neurons suggests that they may integrate visual inputs from the SFGS neuropil layer and relay this information to the deeper
SGC, which is innervated by a subset of retinal afferents and nonvisual afferents from telencephalic and thalamic nuclei (Meek, 1983). To determine if the axonal and dendritic compartments are spatially segregated in bsPVINs we coinjected embryos with $d g 4 i$ and uas:dsred/uas:syp-gfp constructs to visualize the spatial distribution of presynaptic specializations. Although only a fraction of neurons labeled with this construct had adequate expression levels of both fluorescent proteins we were able to image five bsPVIN cells in which we could accurately trace neurite morphology using DsRed and monitor presynaptic specializations using Syp-GFP. In the cell body one or a few bright Syp-GFP puncta were typically observed (Figure $\mathbf{5 A}$ ), which is consistent with previous reports of Syp fusion proteins being sorted through the Golgi complex (Pennuto et al., 2003). In the bsPVIN neurites only the arborization in the deeper SGC layer was observed to contain multiple bright Syp-GFP puncta within varicosities, which have been shown to contain presynaptic active zones (Fletcher et al., 1991). In younger larvae (3-4 dpf) the deeper SGC arbor often contained branches lacking Syp-GFP puncta (see Figure 5A), whereas these were not observed in older larvae (5-8 dpf; see Figure 6). Although this suggests that the deeper arbor is purely axonal we cannot formally exclude the possibility that it may contain both pre- and postsynaptic specializations. In contrast, we never observed bright Syp-GFP puncta in the superficial SFGS arbor. Studies in other cell types have found that Syp-GFP fusion proteins target axonal synapses and are excluded from dendritic compartments (Pennuto et al., 2003), indicating that the SFGS arborization represents the dendritic compartment of the bsPVIN cell type. In summary, the $d g 4 i i$ transgene labels an interneuron type with a dendrite that targets the superficial SFGS layer of the neuropil and an axonal arbor that targets the deeper SGC.

\section{ARRAY TOMOGRAPHIC VALIDATION OF SYP-GFP AS A PRESYNAPTIC MARKER}

Synaptophysin-GFP has been shown to colocalize with various presynaptic proteins in vitro (Bamji et al., 2003; Pennuto et al., 2003). To further validate our finding that presynaptic sites are restricted to the deeper SGC arbor of bsPVIN neurons we performed high-resolution array tomography on a GFP-labeled bsPVIN. A 7-dpf larva containing the GFP-labeled bsPVIN in Figure 6A was fixed and processed for array tomography. Fluorescence images of a single $200 \mathrm{~nm}$ section labeled with an anti-SV2 antibody and DAPI as a nuclear marker (Figures 6B-C) shows that the SV2 label was highly punctate and largely restricted to the tectal neuropil, where it labeled a dense constellation of presumptive synapses. By selecting only SV2 puncta with at least partial overlap with the GFP signal (see Materials and Methods for details) we were able to examine the distribution of SV2containing puncta throughout the bsPVIN arbor (Figure 6D). Analysis of the SV2 immunofluorescent signal within the more superficial SFGS arbor revealed that these puncta were typically not entirely contained within the GFP-labeled process, suggesting these puncta are presynaptic specializations adjacent to the GFP-labeled dendrite (Figure 6G). In contrast, the deeper SGC axonal arbor often contained SV2 puncta that were completely contained within GFP-labeled varicosities (Figures 6E,F). 

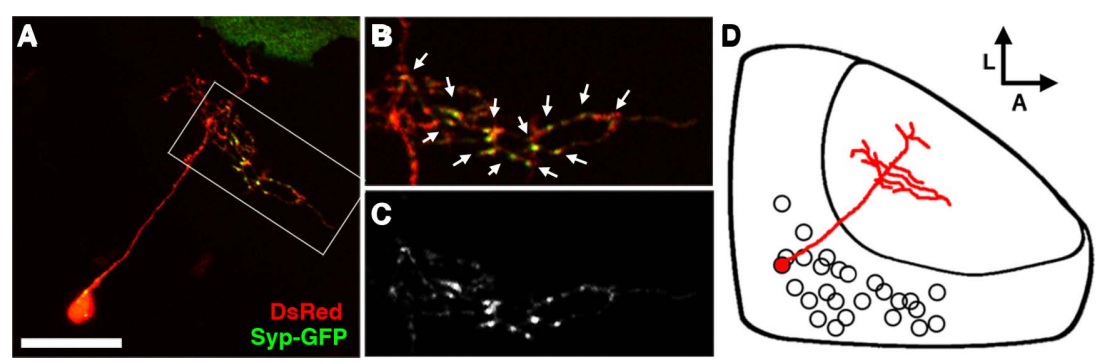

FIGURE 5 |The bistratified periventricular interneuron cell type.

(A) Merged confocal image volume of a single $4 \mathrm{dpf}$ bsPVIN expressing both dsRed (red) and Syp-GFP (green). (B) $2 \times$ magnification of bracketed region in (A). (C) Syp-GFP fluorescence of region in (D). Note the bright Syp-GFP puncta located within the arbor that targets the deeper synaptic layer (arrows in D), whereas puncta are absent from the more superficial arbor (arrowheads in C). (D) Schematic depiction of bsPVIN cell body distribution throughout the SPV layer. Cell traced in red indicates the relative location and scale of neuron depicted in (C). Scale bar, $20 \mu \mathrm{m}$ in (A), $10 \mu \mathrm{m}$ in (B,C).
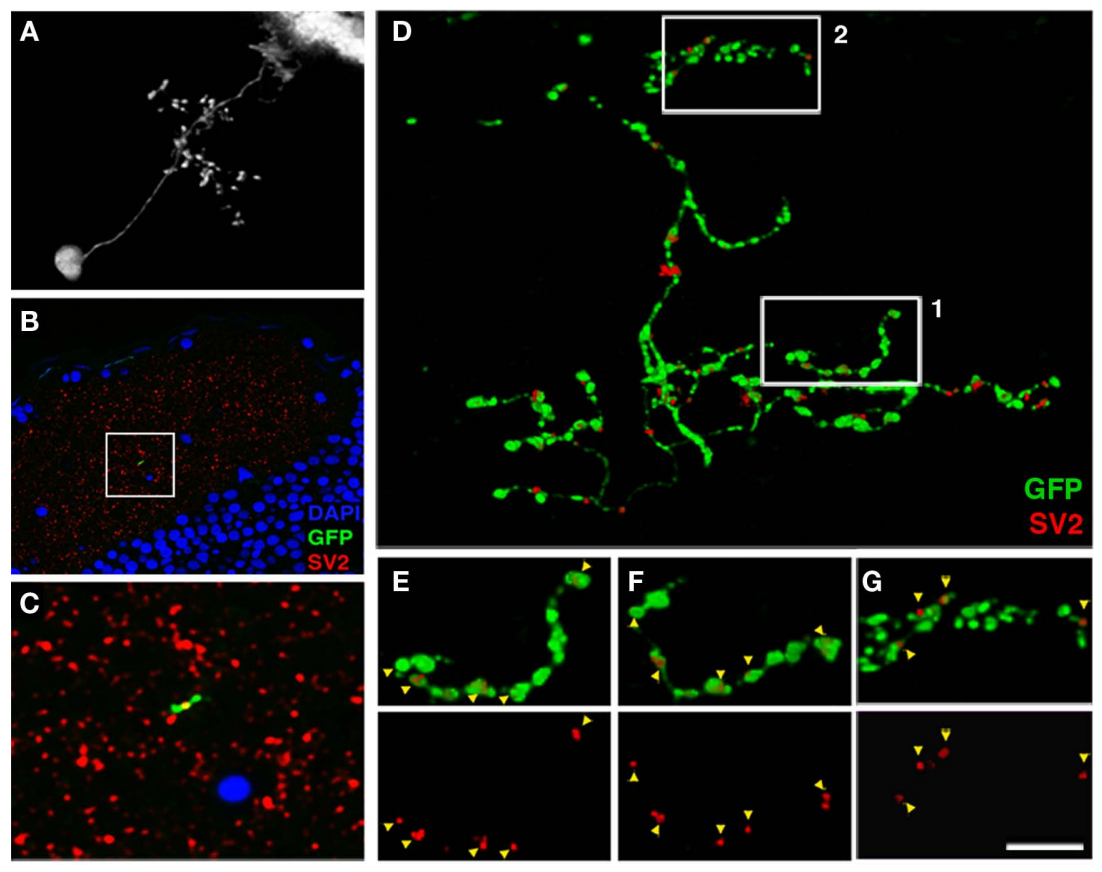

FIGURE 6 | Array tomographic visualization of bsPVIN presynaptic specializations. (A) Individual bsPVIN imaged immediately prior to fixation and processing for array tomographic analysis. (B) Section array was stained with an anti-SV2 antibody (red) and DAPI (blue) to label nuclei. (C) $5 \times$ magnification of boxed region in (B) containing a segment of the SGC targeted axonal arbor of bsPVIN shown in A. Note SV2 puncta contained within segment of GFP-labeled varicosity within this single $200 \mathrm{~nm}$ section. (D) 3D rendering of the section array containing the GFP-labeled neuron in (A-C). Only SV2 puncta that colocalized with areas of GFP labeling were included in rendering. Note large varicosities on the deeper SGC arbor and thin inter-varicosity neurites not detectable by laser-scanning microscopy in (A). (E) 1.75× magnification of boxed region 1 in (D). (F) Forty-five degree rotation of region in (E). Note that a majority of axonal varicosities contain distinct SV2 puncta. (G) $1.75 \times$ magnification of boxed region 2 in (D). Note that the majority of SV2 puncta are not entirely within GFP-labeled dendrite. Scale bar, $20 \mu \mathrm{m}$ in (A), $40 \mu \mathrm{m}$ in (B), $8 \mu \mathrm{m}$ in (C), $3.5 \mu \mathrm{m}$ in (D), $2 \mu \mathrm{m}$ in (E-G).
The improved optical resolution achieved by array tomographic methods revealed that SV2 puncta within axonal varicosities were approximately $250 \mathrm{~nm}$ in diameter, which is consistent with active zone measurements obtained by electron microscopic imaging of the tectum (Meek, 1983). In summary, this array tomography data validates Syp-GFP as a synaptic marker by confirming that endogenous synaptic proteins localize to varicosities on the SGC targeted axonal arbor of bsPVINs.

\section{MORPHOLOGY AND CONNECTIVITY OF THE nSPVIN CELL TYPE}

Of the 92 cells labeled by the $d g 4$ ii plasmid 13 were non-stratified periventricular interneurons (nsPVINs). The low frequency at which these neurons were observed suggests that this cell type may be a small tectal subpopulation. The majority of nsPVINs imaged had cell bodies deep in the SPV layer and were positioned near the medial or posterior edges of the SPV (Figure 7E). Like bsPVINs, nsPVIN cell bodies extend an apical primary 

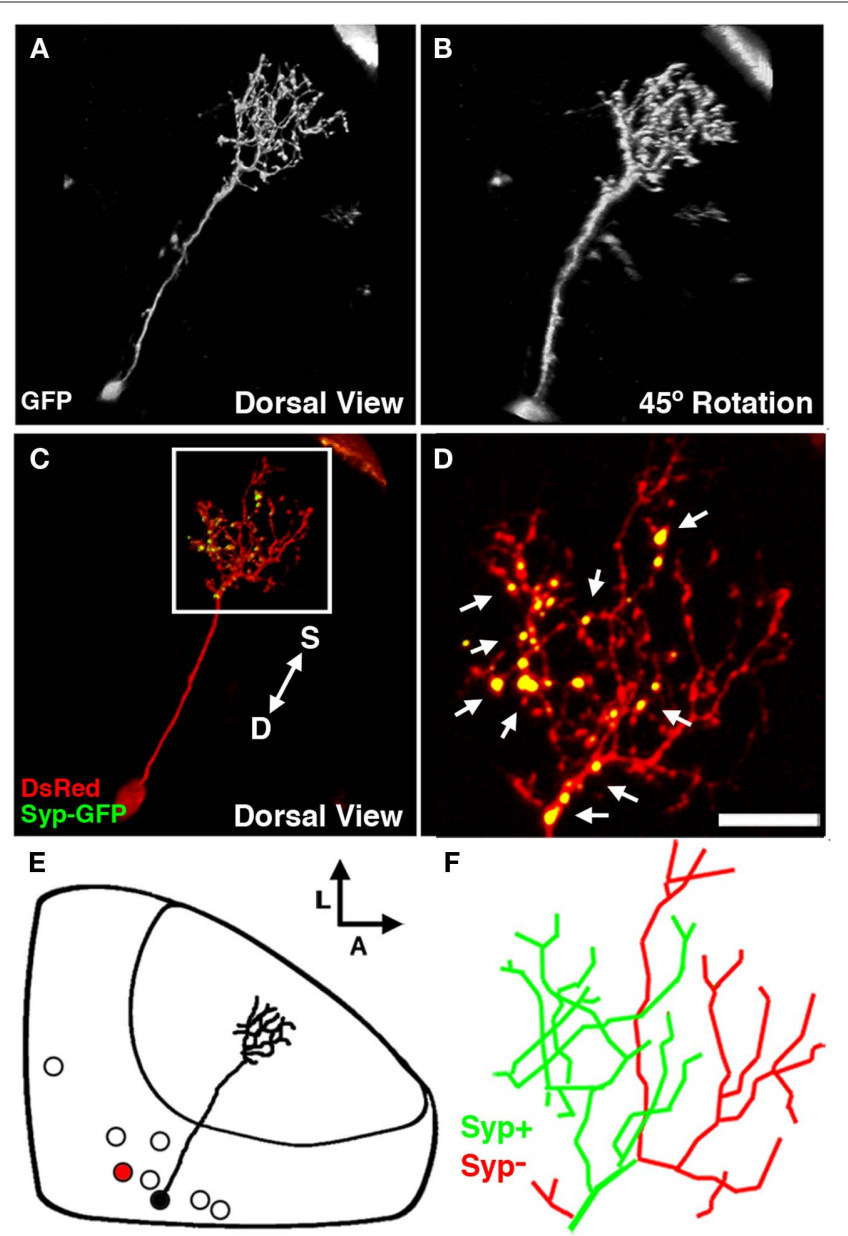

FIGURE 7 |The non-stratified periventricular interneuron cell type. (A) Dorsal view of a two-photon image volume containing a single labeled nsPVIN imaged at $5 \mathrm{dpf}$. Note the long apical neurite extended into the neuropil. (B) Forty-five degree rotation of image volume in (A). Note the absence of any stratification within the arbor. (C) Merged confocal image volume of a single $4 \mathrm{dpf}$ nsPVIN expressing both dsRed (red) and Syp-GFP (green). Arrow depicts orientation of neuropil layers from superficial (S) to deep (D). (D) $2.5 \times$ magnification of boxed region in (C). Note the bright Syp-GFP puncta contained within a subset of neurite branches (arrows). (E) Schematic depiction of nsPVIN cell body distribution throughout the SPV layer. Cell traced in black corresponds to neuron in (A) and (B). Note the deep SPV location of cell body. (F) Manual tracing of the neurite arbor in (D). Green lines indicate branches containing Syp-GFP puncta, whereas branches devoid of Syp-GFP labeling are in red. Scale bar, $20 \mu \mathrm{m}$ in (A-C), $8 \mu \mathrm{m}$ in (D,F).

process into the neuropil and lack an efferent projection. Unlike bsPVINs, these neurons typically possessed a single arborization that lacked laminar specificity (Figures 7A,B). Rotation of 3D image volumes confirmed that the neuronal arbors of nsPVINs are not stratified along any plane and neurite branchpoints are distributed throughout the arbor volume (Figures 7A,B). Based on the average location and thickness of this arbor these neurons primarily target the deeper layer of the SFGS and the SGC. To determine if the axonal and dendritic compartments are spatially segregated in nsPVIN cells we examined the distribution of presynaptic boutons by DsRed/Syp-GFP expression. In five
nsPVIN cells examined there was a clear segregation of Syp-GFP puncta, such that the majority of branches containing Syp-GFP puncta had a deeper position with respect to the neuropil layers, whereas those devoid of Syp-GFP labeling were located more superficially (Figures 7C,D). Detailed analysis of the location of Syp-GFP puncta within these arbors revealed that branches either contained multiple Syp-GFP puncta along their length or were completely devoid of puncta, suggesting that during nsPVIN arbor growth individual branches are specified as either presynaptic or postsynaptic compartments. This possibility is strengthened by the fact that higher order branches retained the synaptic identity of parent branches such that primary axonal branches only gave rise to secondary axonal branches. This can be seen in the traced arbor in Figure 7F, where the axonal and dendritic arbors each arise from two primary branches of the primary apical process.

\section{MORPHOLOGY AND CONNECTIVITY OF THE PVPN CELL TYPE}

A third class of neuron labeled by the $d g 4 i$ ii expression system ( 40 of 92 cells) was a periventricular projection neuron (PVPN) with an apical process that forms a compact arbor in the tectal neuropil that gives rise to a single efferent process (Figure 8A). Unlike the interneurons described above, the cell bodies of projection neurons were primarily restricted to the shallow layers of the cell body layer, typically within $25 \mu \mathrm{m}$ of the boundary between the SPV and the stratum album centrale (SAC; Figure 8E). Based on the average location and thickness of the tectal arbor these neurons target predominantly the deep SGC and SAC layers of the tectal neuropil. Ninety degree rotation of $\mathrm{z}$-stack image volumes along the antero-posterior axis illustrates the trajectory of this efferent projection (Figures 8B,D); it exits from the lateral edge of the tectal neuropil from the SAC and then extends ventrally through the lateral SPV to the underlying diencephalon. Although the ability to clearly trace these efferents was often impeded by labeling of telencephalic and diencephalic neurons with similarly situated processes (arrows in Figure 2), analysis of 12 neurons with efferents that could be clearly traced revealed an average dorso-ventral length of $110.63 \pm 4.93 \mu \mathrm{m}$. Following this ventral extension the process extended posteriorly, forming a sparse axonal arbor with multiple short branches en route to a final termination site in the anterior hindbrain. Within the subset of PVPNs examined the efferent termination site was variable but restricted to the anterior hindbrain within a region spanning from $\mathrm{R} 1$ to $\mathrm{R} 4$. To help determine the exact trajectory of the PVPN projections we examined these efferents in relation to reticulospinal circuitry labeled by pressure injection of the lipophilic tracer DiI into the caudal spinal cord. These experiments revealed that the PVPN efferents follow a trajectory similar to that of the anterior segment of the lateral longitudinal fascicle (LLF; Figure 8F), a large tract positioned lateral to the medial longitudinal fascicle. In the larval brain the LLF extends through the hindbrain, medulla oblongata (MO), and crosses the midbrain-hindbrain boundary to reach the TS. This fascicle is known to contain ascending sensory fibers from the brain stem and spinal cord, descending sensory fibers of trigeminal origin, and processes originating from reticulospinal neurons in the hindbrain reticular formation (Kimmel et al., 1985; Metcalfe et al., 1986; Fame et al., 2006). 


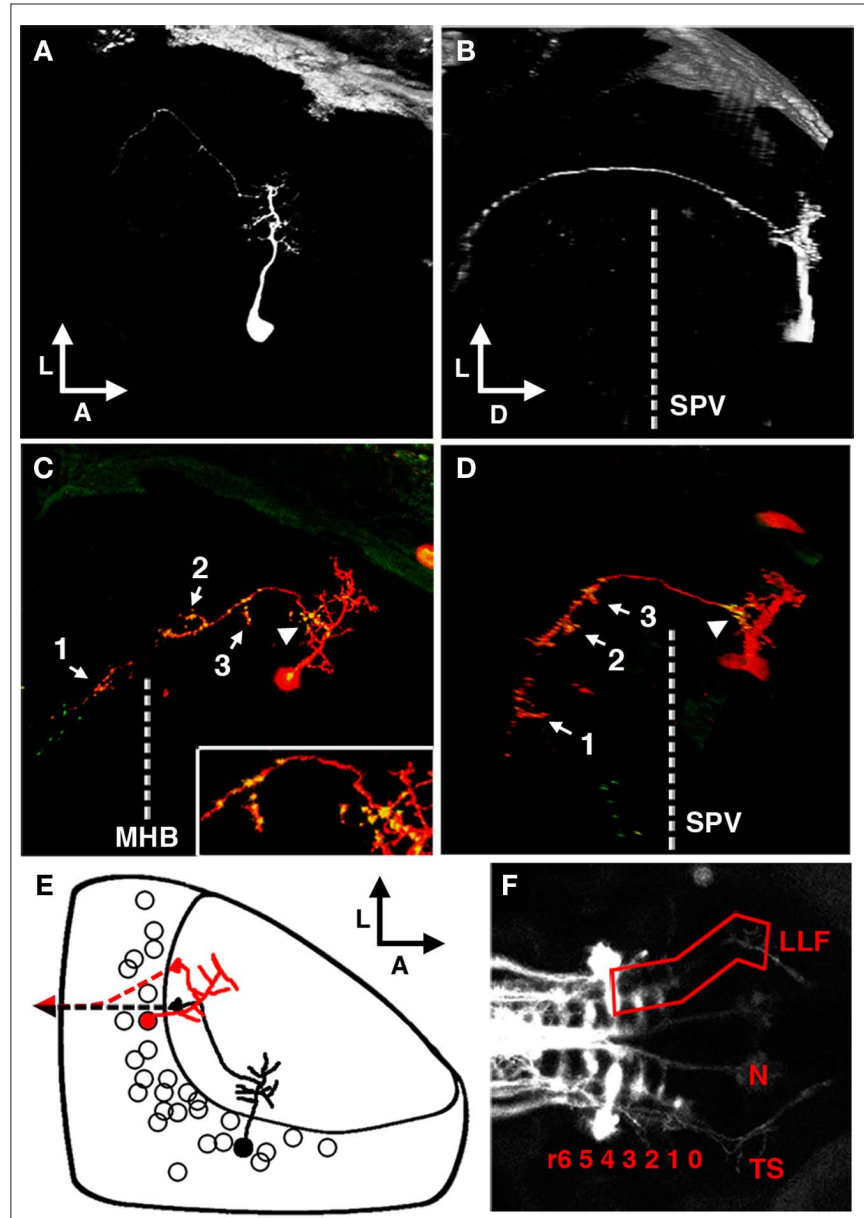

FIGURE 8 |The periventricular projection neuron cell type. (A) Dorsal view of a two-photon image volume containing a single labeled PVPN imaged at $3 \mathrm{dpf}$. Note the short, sparse apical dendrite that gives rise to a single neurite that extends laterally toward the edge of the tectum. (B) Ninety degree rotation of image volume in (A). The single long process has extended ventrally out of the tectum before redirecting to extend in a postero-medial orientation. Dashed line indicates the approximate ventral extent of the SPV. (C) Merged confocal image volume of a single $5 \mathrm{dpf}$ nsPVIN expressing both dsRed (red) and Syp-GFP (green). Dashed line indicates approximate location of the midbrain-hindbrain boundary. Inset: $2 \times$ magnification of region indicated by arrowhead. Note that bright Syp-GFP puncta are located in multiple regions: within the distal segment of the efferent process (arrow 1), the sparse network of collateral branches in the ventral midbrain (arrows 2-3), and a proximal segment within the tectal neuropil (arrowhead). (D) Ninety degree rotation of image volume in (C). (E) Schematic depiction of PVPN cell body distribution throughout the SPV layer. Note that a majority of these cells are located in the shallow SPV close to the neuropil. Cell traced in black correspond to neuron in $(\mathbf{A}, \mathbf{B})$. Note that the more medial cell body location necessitates an initial lateral trajectory in order to reach the edge of the tectal neuropil. In contrast, the cell in (C,D; traced in red) is located laterally, which permits a more direct exit from the tectal neuropil. Dashed line indicates descending axon trajectory after exiting the tectum.

(F) Maximum projection of reticulospinal circuitry labeled by pressure injection of Dil into the spinal cord of a fixed larvae at $5 \mathrm{dpf}$. Boxed region indicates the synaptic field for PVPN efferent axons, which grow along the trajectory of the LLF, which runs from the torus TS, through the $\mathrm{MO}$ to the $\mathrm{HB}$ reticular formation (numbers indicate rhombomere position). Boxed region indicates the typical PVPN arborization field, which spans the TS, MO, and anterior hindbrain. Dashed line indicates midbrain-hindbrain boundary (MHB). Scale bar, $25 \mu \mathrm{m}$ in (A-D), $100 \mu \mathrm{m}$ in (E).
To confirm that this tectofugal process is the axonal compartment we examined the distribution of presynaptic specializations by using the $d g 4 i$ ii plasmid to drive expression of dsRed and Syp-GFP. Syp-GFP puncta were observed in the distal process in the hindbrain (arrow 1 in Figure 8C), suggesting that these neurons form synapses onto neurons with dendrites in the anterior hindbrain. However, in all cases Syp-GFP puncta were also observed within the sparse arbor of axon collaterals formed within the TS and MO (arrows 2 and 3 in Figures 8C,D). This suggests that these projection neurons may have multiple distinct synaptic fields in different brain regions. Surprisingly, in every PVPN imaged we also observed stable Syp-GFP puncta within proximal segments of the axon confined to the tectal neuropil (arrowhead in Figures 8C,D). These proximal Syp-GFP puncta were bright and stable for hours (data not shown), suggesting that they are stable presynaptic specializations and not transport packets being trafficked to the distal axon. These findings suggest that projection neurons in the optic tectum form intermediate synaptic targets within the tectum en route to distal target fields in the TS, MO, and anterior hindbrain.

\section{NEUROTRANSMITTER ANALYSIS OF IDENTIFIED dg4ii NEURON TYPES}

To determine the neurotransmitter phenotype of $d g 4 i$ ii neuron types we conducted retrospective array tomography on fixed larvae following live imaging. As shown in Figure 9, retrospective analysis confirmed that both nsPVINs (four of four cells) and PVPNs (seven of seven cells) were GABAergic, although the need to identify cell types morphologically prior to both sectioning and antibody staining prohibited the analysis of large numbers of neurons. In contrast, every bsPVIN cell analyzed by immunofluorescence was found to be negative for GABA immunoreactivity (six of six cells: data not shown), indicating that this cell type is excitatory, since GABA is the only inhibitory neurotransmitter expressed in the tectum at this developmental stage (Higashijima et al., 2004). During examination of neurotransmitter phenotypes the quality of $v g l u t 2 b$ fluorescence in situ labeling was found to be highly variable (see Materials and Methods). Combined with the need to identify cell types morphologically prior to fixation this prohibited retrospective analysis of vglut $2 b$ expression in individual labeled bsPVINs. However, based on our findings that in the SPV region of $d l x 5 / 6: g f p$ larvae $85.4+5.3 \%$ of cells were GABAergic and $15.6+1.5 \%$ were vglut $2 b$-positive these neurons are most likely glutamatergic.

\section{DISCUSSION \\ dg4ii PLASMID AS A TOOL TO STUDY SPECIFIC CELL TYPES IN THE LARVAL TECTUM}

The nervous system's ability to perform complex, sensory-evoked behaviors develops in parallel with the structural differentiation of neurons and their assembly into functional circuits. Studies examining neuronal development in the tectum have typically utilized methods that label a broad cross-section of tectal neurons (Kaethner and Stuermer, 1997; Niell and Smith, 2005; Sato et al., 2007; Scott and Baier, 2009). Although these studies have revealed general mechanisms common to many different cell types, a complete understanding of tectal circuit function will only be attained when we understand the structure and connectivity of 

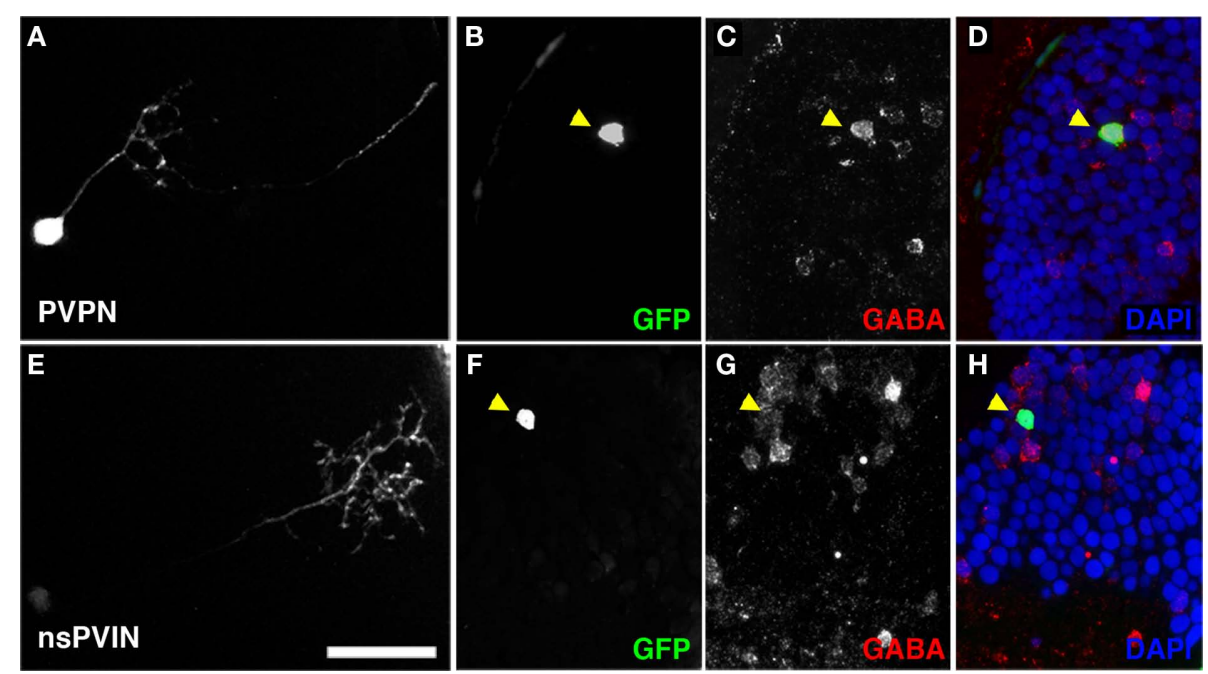

FIGURE 9 | Non-stratified periventricular interneurons and PVPN cell types are GABAergic. (A) Maximum Projection of a single PVPN at $5 \mathrm{dpf}$. $(\mathbf{B}, \mathbf{C})$ Single array section containing cell body of neuron in (A) labeled by GFP fluorescence in (B), anti-GABA immunofluorescence in (C). (D) Merged image of $(\mathbf{B}, \mathbf{C})$ with DAPI nuclear labeling (blue). (E) Maximum Projection of a single
nsPVIN at $5 \mathrm{dpf}$ ( $\mathbf{F , G )}$ Single array section containing cell body of neuron in (E) labeled by GFP fluorescence in (F), anti-GABA immunofluorescence in (G). (H) Merged image of (F,G) with DAPI nuclear labeling (blue). Note preservation of GFP signal and colocalization of GABA immunoreactivity (arrowheads in $\mathbf{D}, \mathbf{H}$ ). Scale bar, $20 \mu \mathrm{m}$. specific neuron types. Our analysis of cell types labeled by the $d g 4 i$ ii plasmid identified three distinct neuron types based on the following criteria: (1) the ability to reliably label these neurons with the dg4ii plasmid, (2) basic morphological features such as arbor size, stratification, and the presence or absence of an efferent axonal projection, (3) the spatial distribution of presynaptic specializations throughout the neuronal arbor, and (4) neurotransmitter phenotype. These criteria allowed us to identify three neuronal cell types with distinct morphological and synaptic features. The following sections will discuss the implications of these findings for current models of tectal circuitry and information processing.

\section{BISTRATIFIED PERIVENTRICULAR INTERNEURONS}

The first tectal cell type discovered using the $d g 4 i$ ii expression system was the bsPVIN: an excitatory interneuron with two stratified arbors that target different layers of the tectal neuropil. In vivo laser-scanning microscopy and retrospective array tomographic imaging of presynaptic protein distribution both confirmed that the axon of this cell targets the SGC neuropil layer, which receives a small subset of retinal afferents. In contrast, the dendrite of this cell targets the SFGS, which is heavily innervated by retinal axons. Interestingly, the axonal and dendritic arbors of these cells exhibited highly precise lamination as indicated by the narrow profile of these arbors in the plane of the synaptic layers. This suggests that anatomically defined synaptic layers (SO, SFGS, SGC, etc.) likely contain functional sublaminae in which corresponding pre- and postsynaptic elements are precisely targeted. This is consistent with the very precise targeting of single retinal axon arbors previously observed in the tectum (Xiao and Baier, 2007).

An interesting feature of the bsPVIN cell type is the fact that they are not inhibitory, as indicated by retrospective single-cell staining with an anti-GABA antibody that labels the majority of neurons labeled in the $d l x 5 / 6: g f p$ stable transgenic. bsPVIN cells are most likely glutamatergic based on the fact that the non-GABAergic population of cells labeled in the $d l x 5 / 6: g f p$ stable transgenic were found to express the glutamatergic marker vglut2b. This is in agreement with previous evidence that the majority of cells in the tectum are either glutamatergic or GABAergic (Higashijima et al., 2004; McLean and Fetcho, 2004; Smear et al., 2007), whereas only a small percentage of cells are cholinergic (Arenzana et al., 2005). Although Dlx family proteins are strong determinants of GABAergic specification in the mouse cortex, it is well established that neurotransmitter specification arises through a combinatorial code of gene expression (Hobert et al., 2010). In many cases generation of GABAergic cells requires induction of gene networks that suppress glutamatergic differentiation (Cheng et al., 2004; Taglialatela et al., 2004; Nakatani et al., 2007; Hori et al., 2008). Therefore it is conceivable that within the combinatorial hierarchy of cell type specification genes there are effectors that act downstream of Dlx family transcription factors to further specify individual cell types with unique structural and functional features. However, it should be emphasized that the focus of this study was to assess the utility of the $d g 4 i i$ expression system to label specific cell types in the larval tectum and further study would be required to confirm that cell populations labeled by $d g 4 i$ ii express Dlx5/6 proteins through the activity of the I56ii enhancer, as recently demonstrated for a population of striatal projection neurons in the mouse (Ghanem et al., 2008).

\section{NON-STRATIFIED PERIVENTRICULAR INTERNEURONS}

The second $d g 4 i i$-positive tectal cell type discovered in this study was the inhibitory nsPVIN, which possesses a single neurite arbor spanning multiple layers of the tectal neuropil. This compact 


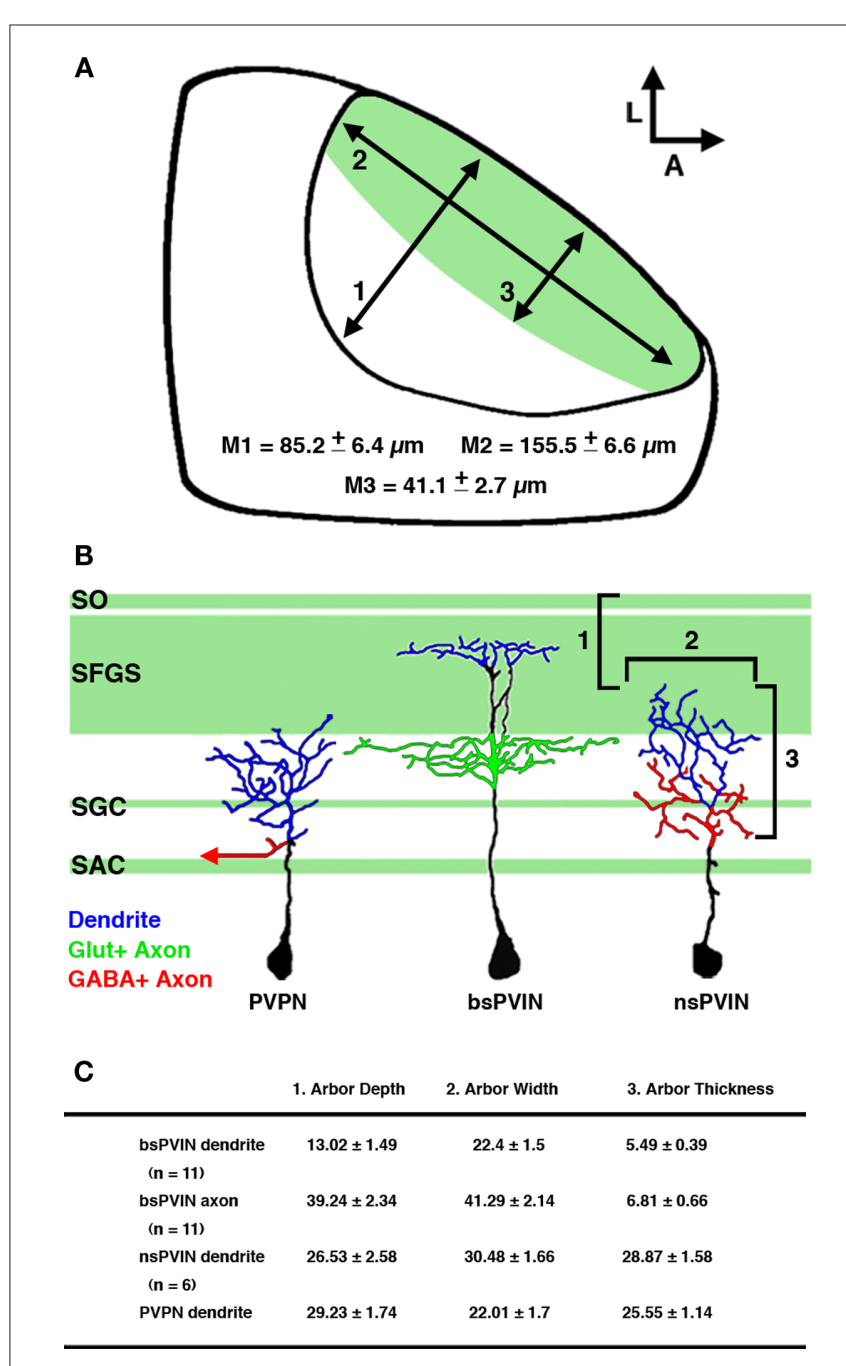

FIGURE 10 | Summary of cell types labeled by the dg4ii plasmid. (A) Schematic of tectal anatomy and the relative sizes of the neuropil and primary retinorecipient laminae in the 5-dpf larvae. Measurements: (1) the maximum length of the tectal neuropil in the plane of the synaptic layers. (2) The maximum thickness of the neuropil in the axis perpendicular to the synaptic layers. (3) The maximum thickness of the primary retinorecipient layers (SO + SFGS). Note that these are maximum measurements at the center of the neuropil and the neuropil thickness is reduced at the edges. (B) Schematic traces of the cell types labeled by the dg4ii expression system and relative position of tectal neurite arbors. Note that nsPVINs an PVPNs primarily arborize in laminae that are primarily non-retinorecipient, whereas bSPVIN dendrites specifically target the SFGS layer. Dendrites are color coded in blue, glutamatergic axons in green (including retinal afferents indicated by green shading), and GABAergic axons in red. (C) Summary of morphological features that distinguish the three cell types identified. Arbor depth indicates the most superficial extent of the arbor as measured from the dorsal surface of the SO. Arbor width is the maximum measured distance in the plane of the synaptic layers. Thickness is the maximum length measured in the axis perpendicular to the synaptic layers. Data expressed as mean \pm SD.

arbor spans layers of both the retinorecipient SFGS and the SGC, which receives only a small fraction of retinal afferents. Live imaging of GFP-synaptophysin to label presynaptic specializations revealed that nsPVIN axons and dendrites exhibit a moderate degree of compartmentalization with respect to the synaptic layers of the tectum. For example, in Figure 7 the nsPVIN axon is mainly located within the deeper synaptic layers, while the dendrites span more superficial layers. Therefore, the dendrites of nsPVINs are more likely to be innervated by retinal axons that target the deeper layers of the SFGS, while their axons are more likely to come into contact with the dendrites of projection neurons. Analysis of the subcellular location of presynaptic puncta within the nsPVIN arbor further suggested that during arbor growth individual branches are specified as either presynaptic or postsynaptic and higher order branches retain the synaptic identity of parent branches. This is in contrast to other neurons lacking segregation of axonal and dendritic compartments, such as olfactory bulb granule cells that mediate lateral inhibition of mitral cells through dendro-denritic synapses (Shepherd et al., 2007). The aforementioned precise lamination of retinal axons and bsPVIN arbors suggests that layers such as the SFGS and SGC are further subdivided into very thin functional sublaminae. Within this context it is interesting that nsPVIN axonal and dendritic arbors may span several functional sublaminae. This may allow nsPVINs to integrate activity from different synaptic layers, which may allow it to integrate different types of synaptic inputs (i.e., visual and non-visual sensory inputs) and transmit this information to multiple postsynaptic cell types. In contrast, cells with precisely stratified axonal and dendritic arbors, such as the bsPVINs, may have a more restricted pool of synaptic partners.

\section{PERIVENTRICULAR PROJECTION NEURONS}

The third $d g 4 i i$-positive cell type identified by this study is an inhibitory projection neuron with a dendritic arbor spanning multiple layers of the tectal neuropil and a long efferent projection that forms a sparse arbor of axon collaterals in the vicinity of the LLF. This target area is in close proximity to the MO, TS, and hindbrain, all of which have been implicated in motor command functions. Live imaging of synaptophysin-GFP to monitor presynaptic boutons confirmed that this tectofugal process is the axon. Interestingly these experiments also revealed that these projection neurons contain stable presynaptic puncta in proximal regions of the axon within the tectal neuropil, suggesting that these projection neurons may also make inhibitory intratectal synapses in addition to inhibiting neurons in other brain areas. This finding is reminiscent of the lateral inhibition mediated by a network of axon collaterals formed by inhibitory projection neurons of the mammalian striatum (Tunstall et al., 2002). Further study will be required to determine the function of these local connections.

Tectal neurons with ipsilateral projections to the hindbrain have been identified in the larval zebrafish by Sato et al. (2007), who used a Brn3a promoter to drive transgene expression. This study determined that these cells were excitatory projection neurons based on the finding that the majority of cells labeled in the brn3a:gfp transgenic were positive for the glutamate transporter vGlut2. However, it should be noted that Brn3a in the mouse mesencephalon is a marker for glutamatergic neurons (Nakatani et al., 2007). If this expression pattern is conserved in the fish this may explain why inhibitory projection neurons are not labeled using the Brn3a promoter. Therefore, it is likely that the PVPNs labeled by the $d g 4 i i$ 
promoter construct are not a subset of the projection neuron pool labeled by the Brn3a promoter. However, these neurons may be a small subset of the cells labeled in the s1038t Gal4 enhancer line (Scott and Baier, 2009), which labels tectal efferents with a projection to the hindbrain and MO. Inhibitory projection neurons have been identified in several systems, well studied examples include pontine omnipause neurons that project to saccade-generating premotor burst neurons in the medullary and midbrain reticular formations (Sparks, 2002), spiny neurons in the striatum that project to the globus pallidus and substantia nigra (Ribak et al., 1976), and superior olive neurons that project to the nucleus magnocellularis (Lachica et al., 1994). This is the first evidence for an inhibitory tectofugal projection in the fish and further study will be required to elucidate the function of this inhibitory output.

\section{IMPLICATIONS FOR TECTAL INFORMATION PROCESSING}

The tectal neuropil can be divided into distinct laminae based on the origin of afferent inputs and structural properties such as the distribution of specific synapse types (reviewed by Meek, 1983). Retinal ganglion cell axons exhibit a precise topographic projection pattern to the tectal neuropil, where they ultimately form synapses onto the dendrites of tectal neurons. In addition to this retinotopic organization, afferent termination sites are also patterned such that the superficial tectal laminae, consisting of the SO and the three SFGS sublayers, receive a vast majority of retinal axons (estimated at $295 \%$; Meek, 1983; Nevin et al., 2010). This connectivity suggests that neurons with postsynaptic elements targeted to these superficial layers likely receive visual synaptic input from retinal afferents and transmit this information to deeper layers of the tectum. A voltage dye imaging study in the tectum of adult trout revealed that stimulation of the optic nerve results in a fast wave of depolarization that spreads through the superficial neuropil layers from anterior to posterior and reflects direct stimulation of retinal axons (Kinoshita et al., 2002). At each point along this wave of depolarization a slower wave of depolarization proceeded from the superficial to deep layers of the tectum that likely corresponds to retinorecipient neurons with radial dendrites receiving visual inputs and transmitting this information to dendrites located deeper in the tectum. The excitatory bsPVIN neuron type identified in this study is an ideal candidate to subserve this superficial to deep information transfer due to its dendrite that targets the superficial layers of the SFGS and an axon that targets the deeper SGC layer. Based on the laminar specificity of bsPVIN dendrites and axons we hypothesize that these neurons function in the transmission of a specific type of visual information from a specialized subset of retinal inputs to projection neurons with dendrites located in the SGC.

In contrast to the bsPVIN cell type, the inhibitory nsPVIN neuron type we identified has a compact arbor that spans the deeper layers of the SFGS and the SGC, suggesting that this neuron could potentially be a synaptic target of bsPVIN neurons. It is possible that these local interneurons mediate intratectal processing that functions to filter the visual information transmitted onto projection neurons. Recent experiments have provided evidence for a similar feed-forward inhibition circuit operating at the superficial layers of the larval tectum (Del Bene et al., 2010). This study characterized superficial interneurons (SINs), a class of inhibi- tory interneurons located near the neuropil surface that extend a broad, stratified arbor that is restricted to the SFGS. These neurons are selectively responsive to large visual stimuli and function to restrict the vertical flow of activity to the deep neuropil layers, thereby functioning as a size selectivity filter that makes the tectal circuit selective for small visual stimuli. Due to the laminar position of bsPVIN dendrites it is possible that these neurons receive excitatory inputs from retinal axons and inhibitory synapses from SINs and function to transmit visually evoked activity to the deeper neuropil layers containing the majority of projection neuron dendrites (see Figure 10C; Scott and Baier, 2009). The inhibitory nsPVIN population identified in this study could function as a feed-forward inhibitory circuit at the deeper layers of the tectum. nsPVINs contain dendritic arbors that span the SGC and only the deepest SFGS sublayer. In the mouse visual system RGC subpopulations with specific response properties have been shown to target specific laminae of the superior colliculus (Kim et al., 2008; Huberman et al., 2009). Therefore it is likely that there are functional differences between the synaptic inputs that target superficial and deep sublayers of the SFGS. Unlike the SFGS, which primarily receives retinal inputs, the SGC layer is known to receive non-visual afferents from telencephalon and thalamus (Meek, 1983), raising the possibility that nsPVINs integrate inputs from non-visual areas and retinal inputs with specific response properties. The role of inhibitory networks in the deeper layers of the tectal neuropil could be to restrict the number of projection neurons activated by a visual stimulus, possibly through modulation by afferent input from brain regions that process non-visual sensory information.

Our identification of an inhibitory projection neuron with efferent targets in the midbrain and hindbrain is the first evidence for an inhibitory tectofugal projection in fish. This inhibitory PVPN population is most likely a very small subset of the total projections from the tectum based on the sparsity of $d l \times 5 / 6: g f p$ axons that exit the tectum compared to the large numbers of tectofugal efferents in this tract labeled by lipophilic dye injection into the tectal neuropil (data not shown). One possibility is that this small population of GABAergic projection neurons serves a highly specialized function within a visually guided behavior such as saccade-generation. Anatomical and microstimulation studies in the adult goldfish indicate that the tectum initiates saccadic eye movements via a prominent tectofugal projection (Luque et al., 2006). In mammals saccade-generation is gated by omnipause neurons that inhibit saccade-generating premotor burst neurons in the medullary and midbrain reticular formations (Sparks, 2002). Several lines of evidence suggest that saccade initiation is driven by the activation of inhibitory inputs onto omnipause neurons (Kamogawa et al., 1996), which are heavily innervated by both glycinergic and GABAergic inputs (Horn et al., 1994). Although OPNs have not been directly identified in larval zebrafish, saccade-generating neurons reside within hindbrain rhombomere 4 (Schoonheim et al., 2010). Therefore, it is possible that the distal axons of $d g 4 i$-positive PVPNs may synapse onto dendrites of saccade-generating cells located within these brain regions. However, future study will be required to determine if this inhibitory tectal projection is a component of saccadegenerating circuitry. 


\section{ACKNOWLEDGMENTS}

We thank Julia Semmelhack, Fumi Kubo, and Tod Thiele for their comments on the manuscript and Marc Ekker and Ryan MacDonald for sharing fish lines and DNA constructs. We also thank Hironobu Ito, Etsuro Ito, Naoyuki Yamamoto,
Ron Meyer, Glenn Northcutt, and Mario Wullimann for their advice regarding fish neuroanatomy. This work was supported by a NSF postdoctoral fellowship to Estuardo Robles as well as NIH R01 NS053358 and a Sandler Opportunity Award to Herwig Baier.

\section{REFERENCES}

Arenzana, F. J., Clemente, D., SanchezGonzalez, R., Porteros, A., Aijun, J., and Arevalo, R. (2005). Development of the cholinergic system in the brain and retina of the zebrafish. Brain Res. Bull. 66, 421 .

Bamji, S. X., Shimazu, K., Kimes, N., Huelsken, J., Birchmeier, W., Lu, B., and Reichardt, L. F. (2003). Role of beta-catenin in synaptic vesicle localization and presynaptic assembly. Neuron 40, 719.

Cheng, L., Arata, A., Mizuguchi, R., Qian, Y., Karunaratne, A., Gray, P. A., Arata, S., Shirasawa, S., Bouchard, M., Luo, P., Chen, C.-L., Busslinger, M., Goulding, M., Onimaru, H., and Ma, Q. (2004). Tlx3 and Tlx1 are post-mitotic selector genes determining glutamatergic over GABAergic cell fates. Nat. Neurosci. 7,510 .

Del Bene, F., Wyart, C., Robles, E., Tran, A., Looger, L., Scott, E. K., Isacoff, E. Y., and Baier, H. (2010). Filtering of visual information in the tectum by an identified neural circuit. Science 330, 669-673.

Fame, R., Brajon, C., and Ghysen, A. (2006). Second-order projection from the posterior lateral line in the early zebrafish brain. Neural Dev. 1, 4 .

Fletcher, T. L., Cameron, P., De Camilli, P., and Banker, G. (1991). The distribution of synapsin I and synaptophysin in hippocampal neurons developing in culture. J. Neurosci. 11, 1617-1626.

Gahtan, E., Tanger, P., and Baier, H. (2005). Visual prey capture in larval zebrafish is controlled by identified reticulospinal neurons downstream of the tectum. J. Neurosci. 25, 9294-9303.

Ghanem, N., Yu, M., Poitras, L., Rubenstein, J. L. R., and Ekker, M. (2008). Characterization of a distinct subpopulation of striatal projection neurons expressing the Dlx genes in the basal ganglia through the activity of the I56ii enhancer. Dev. Biol. 322, 415.

Ghanem, N. L., Jarinova, O., Amores, A., Long, Q., Hatch, G., Park, B. K., Rubenstein, J. L. R., and Ekker, M. (2003). Regulatory roles of conserved intergenic domains in vertebrate dlx bigene clusters. Genome Res. 13, 533-543.

Higashijima, S.-I., Mandel, G., and Fetcho, J. R. (2004). Distribution of prospective glutamatergic, glycinergic, and GABAergic neurons in embryonic and larval zebrafish. J. Comp. Neurol. 480, 1-18.

Hobert, O., Carrera, I., and Stefanakis, N. (2010). The molecular and gene regulatory signature of a neuron. Trends Neurosci. 33, 435.

Hori, K., Cholewa-Waclaw, J., Nakada, Y., Glasgow, S. M., Masui, T., Henke, R. M., Wildner, H., Martarelli, B., Beres, T. M., Epstein, J. A., Magnuson, M. A., MacDonald, R. J., Birchmeier, C., and Johnson, J. E. (2008). A nonclassical bHLH,ÄiRbpj transcription factor complex is required for specification of GABAergic neurons independent of Notch signaling. Genes Dev. 22, 166-178.

Horn, A. K., Buttner-Ennever, J.A., Wahle, P., and Reichenberger, I. (1994). Neurotransmitter profile of saccadic omnipause neurons in nucleus raphe interpositus. J. Neurosci. 14, 2032-2046.

Huberman, A. D., Wei, W., Elstrott, J., Stafford, B. K., Feller, M. B., and Barres, B. A. (2009). Genetic identification of an on-off direction-selective retinal ganglion cell subtype reveals a layerspecific subcortical map of posterior motion. Neuron 62, 327.

Jontes, J. D., Emond, M. R., and Smith, S. J. (2004). In vivo trafficking and targeting of $\mathrm{N}$-cadherin to nascent presynaptic terminals. J. Neurosci. 24, 9027-9034.

Kaethner, R. J., and Stuermer, C. A. O. (1997). Dynamics of process formation during differentiation of tectal neurons in embryonic zebrafish. $J$. Neurobiol. 32, 627.

Kim, I.-J., Zhang, Y., Yamagata, M., Meister, M., and Sanes, J. R. (2008). Molecular identification of a retinal cell type that responds to upward motion. Nature 452, 478 .

Kimmel, C. B., Metcalfe, W. K., and Schabtach, E. (1985). T reticular interneurons: a class of serially repeating cells in the zebrafish hindbrain. J. Comp. Neurol. 233, 365.

Kinoshita, M., Ueda, R., Kojima, S., Sato, K., Watanabe, M., Urano, A., and Ito, E. (2002). Multiple-site optical recording for characterization of functional synaptic organization of the optic tectum of rainbow trout. Eur. J. Neurosci. 16,868.

Koster, R. W., and Fraser, S. E. (2001). Tracing transgene expression in living zebrafish embryos. Dev. Biol. 233, 329.

Lachica, E. A., Rübsamen, R., and Rubel, E. W. (1994). GABAergic terminals in nucleus magnocellularis and laminaris originate from the superior olivary nucleus. J. Comp. Neurol. 348, 403.

Luque, M.A., PÈrez-PÈrez, M. P., Herrero, L., Waitzman, D. M., and Torres, B. (2006). Eye movements evoked by electrical microstimulation of the mesencephalic reticular formation in goldfish. Neuroscience 137, 1051.

Mack-Bucher, J.A., Li, J., and Friedrich, R. W. (2007). Early functional development of interneurons in the zebrafish olfactory bulb. Eur. J. Neurosci. 25, 460-470.

McLean, D. L., and Fetcho, J. R. (2004). Ontogeny and innervation patterns of dopaminergic, noradrenergic, and serotonergic neurons in larval zebrafish. J. Comp. Neurol. 480, 38-56.

Meek, J. (1983). Functional anatomy of the tectum mesencephali of the goldfish. An explorative analysis of the functional implications of the laminar structural organization of the tectum. Brain Res. Rev. 6, 247.

Meek, J., and Schellart, N. A. M. (1978). A golgi study of goldfish optic tectum. J. Comp. Neurol. 182, 89.

Metcalfe, W. K., Mendelson, B., and Kimmel, C. B. (1986). Segmental homologies among reticulospinal neurons in the hindbrain of the zebrafish larva. J. Comp. Neurol. 251, 147.

Meyer, M. P., and Smith, S. J. (2006). Evidence from in vivo imaging that synaptogenesis guides the growth and branching of axonal arbors by two distinct mechanisms. J. Neurosci. 26, 3604-3614.

Micheva, K. D., and Smith, S. J. (2007) Array tomography: a new tool for imaging the molecular architecture and ultrastructure of neural circuits. Neuron 55, 25.

Nakatani, T., Minaki, Y., Kumai, M., and Ono, Y. (2007). Helt determines GABAergic over glutamatergic neuronal fate by repressing Ngn genes in the developing mesencephalon. Development 134, 2783-2793.

Nevin, L., Robles, E., Baier, H., and Scott, E. (2010). Focusing on optic tectum circuitry through the lens of genetics. BMC Biol. 8, 126. doi: 10.1186/1741-7007-8-126

Niell, C. M., Meyer, M. P., and Smith, S. J. (2004). In vivo imaging of synapse formation on a growing dendritic arbor. Nat. Neurosci. 7, 254.
Niell, C. M., and Smith, S. J. (2005). Functional imaging reveals rapid development of visual response properties in the zebrafish tectum. Neuron 45, 941.

Pennuto, M., Bonanomi, D., Benfenati, F., and Valtorta, F. (2003). Synaptophysin I controls the targeting of VAMP2/synaptobrevin II to synaptic vesicles. Mol. Biol. Cell 14, 4909-4919.

Ribak, C. E., Vaughn, J. E., Saito, K., Barber, R., and Roberts, E. (1976). Immunocytochemical localization of glutamate decarboxylase in rat substantia nigra. Brain Res. 116, 287.

Sato, T., Hamaoka, T., Aizawa, H., Hosoya, T., and Okamoto, H. (2007). Genetic single-cell mosaic analysis implicates ephrinB2 reverse signaling in projections from the posterior tectum to the hindbrain in zebrafish. J. Neurosci. 27, 5271-5279.

Schoonheim, P. J., Arrenberg, A. B., Del Bene, F., and Baier, H. (2010). Optogenetic localization and genetic perturbation of saccade-generating neurons in zebrafish. J. Neurosci. 30, 7111-7120.

Scott, E. K., and Baier, H. (2009). The cellular architecture of the larval zebrafish tectum, as revealed by Gal4 enhancer trap lines. Front. Neural Circuits 3:13. doi: 10.3389/neuro.04.013.2009

Scott, E. K., Mason, L., Arrenberg, A. B., Ziv, L., Gosse, N. J., Xiao, T., Chi, N. C., Asakawa, K., Kawakami, K., and Baier, H. (2007). Targeting neural circuitry in zebrafish using GAL4 enhancer trapping. Nat. Methods 4, 323.

Shepherd, G. M., Chen, W. R., Willhite, D., Migliore, M., and Greer, C. A. (2007). The olfactory granule cell: from classical enigma to central role in olfactory processing. Brain Res. Rev. 55, 373.

Smear, M. C., Tao, H. W., Staub, W., Orger, M. B., Gosse, N. J., Liu, Y., Takahashi, K., Poo, M.-M., and Baier, H. (2007). Vesicular glutamate transport at a central synapse limits the acuity of visual perception in zebrafish. Neuron 53, 65.

Sparks, D. L. (2002). The brainstem control of saccadic eye movements. Nat. Rev. Neurosci. 3, 952-964.

Stuhmer, T., Anderson, S. A., Ekker, M., and Rubenstein, J. L. R. (2002). Ectopic expression of the Dlx genes induces glutamic acid decarboxylase and Dlx expression. Development 129, 245-252.

Taglialatela, P., Soria, J. M., Caironi, V., Moiana, A., and Bertuzzi, S. (2004). 
Compromised generation of GABAergic interneurons in the brains of Vax1-/- mice. Development 131, 4239-4249.

Tunstall, M. J., Oorschot, D. E., Kean, A., and Wickens, J. R. (2002). Inhibitory interactions between spiny projection neurons in the rat striatum. $J$. Neurophysiol. 88, 1263.

Westerfield, M. (2000). The Zebrafish Book. A Guide for the Laboratory Use of Zebrafish. Eugene, OR: University of Oregon Press.

Xiao, T., and Baier, H. (2007). Laminaspecific axonal projections in the zebrafish tectum require the type IV collagen Dragnet. Nat. Neurosci. 10, 1529.

Xiao, T., Roeser, T., Staub, W., and Baier, H. (2005). A GFP-based genetic screen reveals mutations that disrupt the architecture of the zebrafish retinotectal projection. Development 132, 2955-2967.
Conflict of Interest Statement: The authors declare that the research was conducted in the absence of any commercial or financial relationships that could be construed as a potential conflict of interest.

Received: 20 October 2010; accepted: 02 February 2011; published online: 22 February 2011.

Citation: Robles E, Smith SJ and Baier $H$ (2011) Characterization of genetically targeted neuron types in the zebrafish optic tectum. Front. Neural Circuits 5:1. doi: 10.3389/fncir.2011.00001

Copyright (C) 2011 Robles, Smith and Baier. This is an open-access article subject to an exclusive license agreement between the authors and Frontiers Media SA, which permits unrestricted use, distribution, and reproduction in any medium, provided the original authors and source are credited. 